\title{
ACANTOSE NIGRICANS COMO SÍNDROME PARANEOPLÁSICA: ASPECTOS CLÍNICOS
}

\author{
Inêz Gabrielle Duarte Sousa ${ }^{1}$, Daniela Matos Carneiro ${ }^{1}$, \\ Paula Gabriella de Sousa Araújo ${ }^{1}$, Maria Mirelle Ferreira Leite Barbosa ${ }^{1}$, Filipe Rolim Medeiros ${ }^{1}$, \\ Rafaela Matos Carneiro ${ }^{2}$, Millene Ivania Ferreira Leite Barbosa ${ }^{3}$.
}

Introdução: A Acantose Nigricans (AN) é caracterizada pela presença de placas acastanhadas, simétricas, não pruriginosas nas dobras da pele e na parte de trás do pescoço que podem infiltrar e se apresentar como placas hiperqueratóticas. Já a Síndrome Paraneoplásica é uma condição que surge em associação a uma malignidade em outro lugar do corpo, mas, por si só, não é cancerígena. Objetivo: Revisar as evidências das características clínicas da acantose nigricans do tipo maligna. Método: Trata-se de uma revisão integrativa da literatura com levantamento de dados no Portal Regional da Biblioteca Virtual em Saúde - BVS utilizando os unitermos do DeCS - Descritores em Ciências da Saúde "Paraneoplastic syndromes", "Acanthosis nigricans" e "Neoplasms". Foram incluídos relatos de casos publicados entre 2008-2017, em inglês, que tinham como assunto principal "Acantose Nigricans" e que permitiam acesso ao texto completo. Dos 12 artigos encontrados, 9 foram selecionados conforme adequação à proposta deste trabalho. Resultados: A AN é causada por fatores que estimulam a proliferação de queratinócitos epidérmicos e fibroblastos dérmicos. Pode se apresentar de forma benigna ou maligna. A forma benigna está associada à resistência à insulina, obesidade e uso de alguns medicamentos enquanto a maligna está associada à tumores. Apesar da $\mathrm{AN}$ maligna ser rara vários tipos de cânceres tem sido relatados, nos quais os intraabdominais são os mais comuns, particularmente, o adenocarcinoma gástrico. A AN maligna é clinicamente indistinguível das formas benignas, entretanto, é importante desconfiar quando as lesões surgem rapidamente, são extensas, sintomáticas, vistas em locais atípicos ou apresentam rápida progressão. Ademais, pode coexistir com outros marcadores cutâneos de malignidade como o sinal de Leser Trelat, hipertricose lanuginosa, "palmas em tripa" e papilomatose florida cutânea. Finalmente, a AN pode desaparecer com o tratamento da malignidade e reaparecer no caso de recorrência ou metástase. Conclusão: As alterações cutâneas podem ser pistas importantes para o diagnóstico de malignidade e o reconhecimento precoce da sinalização de paraneoplasia cutânea é de grande valor para prevenir a progressão da neoplasia. Dessa forma, qualquer caso de AN incomum deve ser investigado de forma vigorosa afim de procurar tumor subjacente, pois o diagnóstico oportuno e o tratamento adequado podem resultar em um desfecho menos desfavorável ao paciente.

Palavras-chave: paraneoplastic syndromes, acanthosis nigricans, neoplasms.

\footnotetext{
${ }^{1}$ Acadêmico do curso de Medicina da Faculdade de Medicina Estácio de Juazeiro do Norte ;

${ }^{2}$ Enfermeira graduada pela Universidade Regional do Cariri - URCA (CE);

${ }^{3}$ Cirurgiã Geral formada pelo Hospital Getúlio Vargas - HGV - Recife (PE); Autor Correspondente: inez.gabrielle01@gmail.com.
}

22 Id on Line Rev. Mult. Psic. V.12, N. 40. 2018 - ISSN 1981-1179 EDIÇÃO ESPECIAL: I CURSO DE ONCOLOGIA DO CARIRI / II JORNADA DE PESQUISA QUANTI-QUALITATIVA EM ONCOLOGIA. JUAZEIRO DO NORTE, 05 A 10 DE MARÇO DE 2018. Edição eletrônica em http://idonline.emnuvens.com.br/id 